\title{
Early changes in macular optical coherence tomography parameters after Ranibizumab intravitreal injection
} in patients with exsudative age-related macular degeneration

\author{
Nicole Antunes de Almeida and Osias Francisco de Souza*
}

\begin{abstract}
Background: Evaluation of the impact of different macular optical coherence parameters on visual acuity as early as 1 day after injection of ranibizumab in patients with subfoveal exsudative age-related macular degeneration.

Methods: This was an interventional, non randomized, open label prospective study, where we evaluated 20 eyes of 20 patients affected by exudative age-related macular degeneration. These patients were treated with injections of ranibizumab between February 2013 and January 2015. The primary endpoint of this study was to evaluate the early changes in optical coherence tomography parameters (retinal thickness, central and total retinal volume) and impact on best-corrected visual acuity (BCVA) obtained by logarithm of minimum resolution using ETDRS protocol in patients treated with a single dose intravitreal injection of ranibizumab $(0.5 \mathrm{mg} / 0.05 \mathrm{~mL})$ during the first month of follow. The patients were evaluated on the first day, them at 7 and 30 days after the treatment. The National Eye Institute Visual Functioning Questionnaire was applied during the study period to assess early perception of ranibizumab injection effectiveness. The adverse events were monitored throughout the study.
\end{abstract}

Results: Central retinal thickness values at $1(464.0 \pm 97.8 \mu \mathrm{m}), 7(379.9 \pm 107.8 \mu \mathrm{m})$ and 30 days $(365.5 \pm 95.1 \mu \mathrm{m})$ after ranibizumab injection showed a statically significant reduction when compared with baseline results $(P=0.01$, $P=0.001, P=0.001$, respectively). Similar alterations were observed in central and total retinal volume, which were detected early on the first day of evaluation, after the measurement at baseline (central: $0.36 \pm 0.07 \mathrm{vs}$. $0.40 \pm 0.10 \mathrm{~mm}^{3}, P=0.01$; total: $9.62 \pm 1.10$ vs. $\left.9.99 \pm 2.56 \mathrm{~mm}^{3}, P=0.002\right)$ and remained steady at $7(P=0.001$, $P=0.002$, respectively) and 30 days $(P=0.001, P=0.004$, respectively) with slight variations without losing their gains in these parameters. The best-corrected logarithm of minimum angle of resolution (logMAR) showed a statistically significant difference when compared to the baseline. ( $0.81 \pm 0.16$ vs. $0.67 \pm 0.24, P=0.005)$. The NEI-VFQ-25 questionnaire demonstrate statically significant results after treatment. When patients were asked about the subjective improvement in visual quality, over $80 \%$ reported early improvement. Throughout the period of follow-up visits, no serious adverse events were reported.

Conclusion: Intravitreal injection of ranibizumab can produce early changes in optical coherence tomography parameters and an improvement in perceived visual quality of patients with subfoveal exsudative age-related macular degeneration.

\footnotetext{
*Correspondence: osiasfs@uol.com.br

Centro Médico de Oftalmologia/ProRetina CAPC - University of Campinas

School of Medical Sciences, Rua Engenheiro Carlos Stevenson 66,

Campinas, SP 13092132, Brazil
} 
Keywords: Age-related macular degeneration, Optical coherence tomography, Ranibizumab, Subfoveal exsudative, Choroidal neovascularization

\section{Background}

Age-related macular degeneration (AMD) is a common cause of central visual loss and legal blindness in older adults. It is most common in individuals over the age of 65 years, occurring in approximately $25 \%$ in this age group [1]. The two most common forms of AMD are known as dry (i.e. atrophic) and wet (i.e. subfoveal exsudative). The exudative form is characterized by growth of choroidal neovascularization (CNV), causing hemorrhagic or serous subretinal and/or pigment epithelium detachment and is responsible for over $90 \%$ of cases of severe visual loss [1-3].

$\mathrm{CNV}$ development involves a series of factors such as inflammation and angiogenesis, leading eventually to sub retinal fibrosis. Vascular endothelial growth factor (VEGF) has been considered an important factor in the development mechanism of CNV. Different treatments targeting VEGF have shown improvement of vision in subfoveal exsudative AMD patients and VEGF inhibition is now considered as an effective strategy for the treatment of this condition [4-6].

Ranibizumab is a high-affinity recombinant monoclonal antibody fragment (Fab) that neutralizes all biologically active isoforms of VEGF-A. It was approved by the FDA (Food and Drug Administration) in June 2006 for treatment of all subtypes of neovascular AMD and it has been proven clinical effective in improving mean visual acuity in several clinical trials [5, 7]. Many diagnostic modalities can be used for detection of CNV in AMD patients. Time domain and spectral-domain optical coherence tomography (SD-OCT) and fluorescein angiography (FA) still remain the best methods to detect CNV [8].

Quantitative OCT analysis, a non-invasive imaging technique, is used to track treatment outcomes in patients with subfoveal exsudative AMD and may play an increasingly important clinical role in the development of anti-VEGF therapies. There are few studies available that have attempted to systematically examine the correlation between OCT-derived morphologic parameters and visual acuity. However, previous studies have reported significant early visual and OCT benefits (within 7 days and a month, respectively) after treatment with an anti-VEGF agent (bevacizumab), even with a low resolution technique. Those studies however used time domain OCT, a technology with lower resolution and poor reproducibility of radial scans, so the information about retinal and subretinal morphology is limited [9-11].
Considering the importance of evaluating the impact of different OCT parameters on visual acuity in the first week after injection of ranibizumab in AMD patients, we used an equipment with SD-OCT capability to obtain images to define which of these parameters correlates better with visual acuity and prognosis [12-14]. Furthermore, since there is no data about patients' perception of the effectiveness of ranibizumab at an early stage of treatment, we applied the National Eye Institute Visual Functioning Questionnaire (NEI-VFQ-25) during treatment [15].

\section{Methods}

This trial was an interventional, non randomized, open label prospective study with a single arm of treatment. The study and informed consent were approved by the local ethics committee at Medical School of the State University of Campinas. Patients older than 50 years of age, with BCVA between 20/40 and 20/320, determined by ETDRS chart, and primary choroidal neovascularization associated with AMD (type 2) were included. Patients who had other ocular disorders/procedures that could confound interpretation of results, or had reasonable condition that contraindicate the use of this investigational drug, who required ocular surgical procedures during 12 month study period, or needed concomitant therapy with topical ocular or systemically corticosteroids within 4 months prior, were excluded. Previous treatment with anti-angiogenic drugs (pegaptanib sodium, anecortave acetate, bevacizumab, ranibizumab, etc.), known hipersensitivity to ranibizumab and any component of formulation, or inability to comply with study/follow-up procedures, were also considered exclusion criteria.

To establish a diagnosis of active choroidal neovascularization, the presence of leakage would have to be detected by fluorescein angiography test, and the fluid observed either inside or below the retina by SD-OCT. After discussion of the pathogenesis of AMD, the alternatives to therapy, potential risks of treatment with ranibizumab, and prior to receiving the study drug injection, all patients who were considered eligible gave consent for the use of their data for research purposes.

The participants were also asked to complete the NEIVFQ-25 form for assessment of the visual impairment and symptoms in order to verify the influence of treatment on improving visual impairment and visual symptoms such as the emotional and visual function. This form 
is a reliable and validated instrument, especially useful in settings such as clinical trials where interview length is a critical consideration. The NEI-VFQ-25 includes 25 core items to measure 12 domains of vision function. In this trial, we used a previously validated Portuguese version of the NEI-VFQ-25, which was applied at the baseline visit, and at 1,7 , and 30 days after administration.

Prior to drug injection, the BVCA for each studied eye was obtained by LogMAR using the ETDRS visual acuity protocol and the presence of leakage from CNV involving the fovea was confirmed by the FA test. Central retinal thickness was assessed twice to $1 \mathrm{~mm}$ from the center circle using 7 lines scan protocol and dense protocol using high resolution SD-OCT (Heidelberg Spectralis, version 5.1, Germany). Line scanning images were also used for examine morphological features of retinal including cystoid spaces, retinal thickening, sub retinal fluid or pigment epithelial detachment.

After preparation of the eyelids with a $10 \%$ povidoneiodine swab and instillation of $5 \%$ povidone-iodine in the lower conjunctival fornix, the eye was anesthetized with topical $1 \%$ tetracaine chloridrate. A lid speculum was used for access to the eye with minimal pressure on the globe and $0.5 \mathrm{mg} / 0.05 \mathrm{~mL}$ of ranibzumab (Lucentis ${ }^{\circledR}$, Novartis Pharma AG, Switzerland) was injected intravitreally with a 30-gauge needle followed by administration of gatifloxacin ophthalmic solution. The permeability of the central retinal artery was verified by ophthalmic assessment and the intraocular pressure expected before discharge of a participant was $<30 \mathrm{mmHg}$. Participants received instructions for the administration of a topical antibiotic for a period of 5 days after this procedure.

In order to evaluate change in study parameters, the data collected included a full ophthalmological exam, BCVA and retinal imaging with quantitative SDOCT parameters (foveal thickness, foveal volume-central $1 \mathrm{~mm}$ circle, retinal volume at 3 and $6 \mathrm{~mm}$ central circles), and the completion of the NEI-VQF-25.

The software programms used for statistical analysis were SPSS V.17 (DMSS Software ${ }^{\circledR}$-São Paulo-Brasil), Minitab 16 (Minitab Inc. USA) and Microsoft Excel (2010). We considered the significance level of 0.05 (5\%) with a confidence interval of 95\% (CI 95\%) for this study. A non--parametric test (Wilcoxon, Friedman and Equality test) was chosen due to the small sample (less than 30 individuals).

\section{Results}

Twenty eyes of 20 consecutively participants (11 women and 9 men), with a mean age of $76.1 \pm 8.7$ years and a clinical diagnosis of exudative AMD were selected for this trial. All patients selected were submitted to a
Ranibizumab intravitreal injection between February 2013 and January 2015 (Table 1).

The changes in BCVA log MAR values from baseline to 1,7 and 30 days after injection are shown in Table 2 . The comparison with the baseline values $(0.81 \pm 0.16)$ showed a gradual increase in BCVA observed after ranibizumab intravitreal injection $(0.72 \pm 0.23$ at day 7$)$, becoming even more evident after 30 days $(0.67 \pm 0.24)$ with statistically significant difference $(P=0.005)$.

A month after ranibizumab injection the values obtained for central retinal thickness showed a significant decrease, as a consequence of reduction of subfoveal fluid (under internal retina). The retinal thickness values started at $524.5 \mu \mathrm{m}$ and decrease to $353.0 \mu \mathrm{m}$ on day 30 $(P=0.001)$ (Table 3$)$.

Immediate decrease in central retina volume (CRV) $\left(0.41-0.36 \mathrm{~mm}^{3}\right)$ and total retinal volume (TRV) (9.94$9.34 \mathrm{~mm}^{3}$ ) were seen early on the first day of evaluation and reduced more at days 7 and 30 with minor variations (Tables 4 and 5).

Although of NEI-VFQ-25 questionnaire results did not demonstrate significant change before and after treatment, except when asked about the subjective improvement in visual quality, over $80 \%$ of patients reported early improvement.

Intraocular pressure was measured before drug administration and after 7 days (T0, T7 and T30). No clinically significant change from baseline can be observed in IOP values $(P=0.062)$. Throughout the period of follow-up visits, no serious adverse events were reported.

\section{Discussion}

Symmetrical distribution and a small variability (variation coefficient $<50 \%$ ) in the population was considered a positive point for results validation, as demonstrated in the demographic data.

Overall, treatment with ranibizumab provided clinically and statistically significant improvement in subfoveal exsudative AMD [15-17]. Corroborating others, our results of changes in the SD-OCT parameters and the

\section{Table 1 Demographic characteristics}

\begin{tabular}{lll}
\hline & Characteristics & Result \\
\hline 1 & No of eyes & 20 (one of each participant) \\
2 & Age (mean \pm SD) & $76.1 \pm 8.7$ years; IC $(72.3 ; 79.9)$ \\
& Age (median) & 75.5 (range 55-93 years) \\
3 & Gender & 11 women, 9 men \\
4 & Eye & 14 OD, 06 OS \\
5 & Characteristic of crystalline lens & 15 IOL, 05 translucent \\
6 & PED & 09 no, 11 yes \\
7 & Presence of drusen & 3 no, 17 yes \\
\hline
\end{tabular}

$I O L$ intraocular lens, $P E D$ pigment epithelial detachment, $O D$ right eye, OS left eye 
Table 2 The changes in BCVA/LogMAR measurements

\begin{tabular}{lllll}
\hline & Time after injection & Mean \pm SD & IC95\% & $P$ value \\
\hline 1 & Baseline (prior injection—T0) & $0.81 \pm 0.16$ & $0.74 ; 0.88$ & - \\
2 & 1 day after (T1) & $0.76 \pm 0.19$ & $0.67 ; 0.85$ & $0.066^{\mathrm{a}}$ \\
3 & 7 days after (T7) & $0.72 \pm 0.23$ & $0.61 ; 0.83$ & $0.016^{\mathrm{b}}$ \\
& & & & $0.157^{\mathrm{C}}$ \\
4 & \multirow{2}{*}{ 30 days after (T30) } & $0.67 \pm 0.24$ & $0.55 ; 0.78$ & $0.005^{\mathrm{d}}$ \\
& & & & $0.028^{\mathrm{e}}$ \\
& & & & $0.109^{\mathrm{f}}$ \\
\hline
\end{tabular}

${ }^{\mathrm{a}} \mathrm{TO} / \mathrm{T} 1 ;{ }^{\mathrm{b}} \mathrm{TO} / \mathrm{T7} ;{ }^{\mathrm{C}} \mathrm{T} 1 / \mathrm{TT} ;{ }^{\mathrm{d}} \mathrm{TO} / \mathrm{T} 30 ;{ }^{\mathrm{e}} \mathrm{T} 1 / \mathrm{T} 30 ;{ }^{\mathrm{f}} \mathrm{T} 7 / \mathrm{T} 30$

Table 3 Changes in central retinal thickness over a month $(n=20)$

\begin{tabular}{|c|c|c|c|c|}
\hline & Time of injection & Mean $\pm \mathrm{SD}(\mu \mathrm{m})$ & Median $(\mu \mathrm{m})$ & $P$ value \\
\hline 1 & $\begin{array}{l}\text { Baseline (prior injec- } \\
\text { tion-T0) }\end{array}$ & $512.3 \pm 126.9$ & 524.5 & - \\
\hline 2 & $\begin{array}{l}1 \text { day after injection } \\
\text { (T1) }\end{array}$ & $464.0 \pm 97.8$ & 458.5 & $0.014^{\mathrm{a}}$ \\
\hline 3 & $\begin{array}{l}7 \text { days after injection } \\
\text { (T7) }\end{array}$ & $379.9 \pm 107.8$ & 351.0 & $\begin{array}{l}0.001^{b} \\
0.001^{c}\end{array}$ \\
\hline 4 & $\begin{array}{l}30 \text { days after injec- } \\
\text { tion (T30) }\end{array}$ & $365.5 \pm 95.1$ & 353.0 & $\begin{array}{l}0.001^{d} \\
0.001^{e} \\
0.179^{f}\end{array}$ \\
\hline
\end{tabular}

${ }^{\mathrm{a}} \mathrm{TO} / \mathrm{T} 1 ;{ }^{\mathrm{b}} \mathrm{TO} / \mathrm{T7} ;{ }^{\mathrm{C}} \mathrm{T} 1 / \mathrm{TT} ;{ }^{\mathrm{d}} \mathrm{T} 0 / \mathrm{T} 30 ;{ }^{\mathrm{e}} \mathrm{T} 1 / \mathrm{T} 30 ;{ }^{\mathrm{f}} \mathrm{T} 7 / \mathrm{T} 30$

Table 4 Change in central retinal volume over a month by SD-OCT $(n=20)$

\begin{tabular}{lllll}
\hline & Time of injection & $\begin{array}{l}\text { Mean } \pm \text { SD } \\
\left(\mathbf{m m}^{\mathbf{3}}\right)\end{array}$ & Median $\left(\mathbf{m m}^{\mathbf{3}}\right)$ & $\boldsymbol{P}$ value \\
\hline 1 & $\begin{array}{c}\text { Baseline (prior } \\
\text { injection-T0) }\end{array}$ & $0.40 \pm 0.10$ & 0.41 & - \\
2 & $\begin{array}{l}1 \text { day after injec- } \\
\text { tion (T1) }\end{array}$ & $0.36 \pm 0.07$ & 0.36 & $0.011^{\mathrm{a}}$ \\
3 & $\begin{array}{l}\text { 7 days after injec- } \\
\text { tion (T7) }\end{array}$ & $0.29 \pm 0.07$ & 0.28 & $0.001^{\mathrm{b}}$ \\
4 & $\begin{array}{l}\text { 30 days after injec- } \\
\text { tion (T30) }\end{array}$ & $0.29 \pm 0.08$ & 0.28 & $0.003^{\mathrm{c}}$ \\
& & & $0.001^{\mathrm{d}}$ \\
& & & $0.002^{\mathrm{e}}$ \\
& & & $0.265^{\mathrm{f}}$
\end{tabular}

${ }^{\mathrm{a}} \mathrm{TO} / \mathrm{T} 1 ;{ }^{\mathrm{b}} \mathrm{T} 0 / \mathrm{T} 7 ;{ }^{\mathrm{c}} \mathrm{T} 1 / \mathrm{T} 7{ }^{\mathrm{d}} \mathrm{T} 0 / \mathrm{T} 30 ;{ }^{\mathrm{e}} \mathrm{T} 1 / \mathrm{T} 30 ;{ }^{\mathrm{f}} \mathrm{T} 7 / \mathrm{T} 30$

visual acuity in patients with AMD after the first intravitreal injection of ranibizumab $(0.5 \mathrm{mg})$ demonstrated a statistically significant difference over 30 days, which may be verified by the reduced values of the retina thickness and lowering the total volume of the central retina.

However, we also found statistically significant difference in the days 1 and 7. Pharmacokinetic studies about anti-vegf described relatively long half-lives (7-10 days)
Table 5 Change in total retinal volume over a month by SD-OCT $(n=20)$

\begin{tabular}{|c|c|c|c|c|}
\hline & Time of injection & $\begin{array}{l}\text { Mean } \pm S D \\
\left(\mathrm{~mm}^{3}\right)\end{array}$ & Median $\left(\mathrm{mm}^{3}\right)$ & $P$ value \\
\hline 1 & $\begin{array}{l}\text { Baseline (prior } \\
\text { injection-T0) }\end{array}$ & $9.99 \pm 2.56$ & 9.94 & - \\
\hline 2 & $\begin{array}{l}1 \text { day after injec- } \\
\text { tion (T1) }\end{array}$ & $9.62 \pm 1.10$ & 9.34 & $0.002^{\mathrm{a}}$ \\
\hline 3 & $\begin{array}{l}7 \text { days after injec- } \\
\text { tion (T7) }\end{array}$ & $8.77 \pm 0.83$ & 8.48 & $\begin{array}{l}0.002^{b} \\
0.001^{c}\end{array}$ \\
\hline 4 & $\begin{array}{l}30 \text { days after injec- } \\
\text { tion (T30) }\end{array}$ & $8.75 \pm 1.39$ & 8.30 & $\begin{array}{l}0.004^{d} \\
0.028^{e} \\
0.391^{f}\end{array}$ \\
\hline
\end{tabular}

${ }^{a} \mathrm{TO} / \mathrm{T} 1 ;{ }^{b} \mathrm{TO} / \mathrm{T} 7 ;{ }^{\mathrm{c}} \mathrm{T} 1 / \mathrm{TT} ;{ }^{\mathrm{d}} \mathrm{TO} / \mathrm{T} 30 ;{ }^{\mathrm{e}} \mathrm{T} 1 / \mathrm{T} 30 ;{ }^{\mathrm{f}} \mathrm{T} 7 / \mathrm{T} 30$

after intravitreal depot injections and clinical durations of action that usually exceed 4 weeks $[16,18]$. Therefore, it is not the peak level of drug that will dictate efficacy after 1 month, but rather the amount of residual drug present in the eye 1 month after the injection, which is also known as the trough level [17]. It seems reasonable to assume that these eyes may initially respond well.

Despite the importance of these findings, the primary aim of this study was to access the early changes in volume and retinal thickness using SD-OCT and its possible impact on visual acuity. LogMAR BCVA results showed a significant improvement within the first day and 1 month after only one ranibizumab intravitreal injection $(P<0.05)$. In this context it was possible to observe the immediate effect of ranibizumab in reducing thickness and volume of retina, which started early (day 1) and persisted through days 7 and 30 with slight variations.

The persistence of these effects in reducing the thickness and volume of the retina suggests that a clinician could utilise Ranibizumab intravitreal injections on a 30 days interval as a safe protocol for AMD.

Also, in a phase I/II study with neovascular AMD patients, a repeated treatment with intravitreal injections of ranibizumab for up to 3 months were associated with an improvement in visual acuity and decreased leakage from choroidal neovascularization in these population, corroborating the hypothesis that a long-term evaluation with repeated doses of drugs could demonstrate better results $[19,20]$.

The NEI-VFQ-25 questionnaire results demonstrate significant alteration before and after treatment when patients were asked about the subjective improvement in visual quality. The results also showed that over $80 \%$ of patients reported early improvement as a high motivation to continue treatment. 


\section{Conclusion}

In the present study we observed that a single intravitreal injection of ranibizumab can produce significant early changes in SD-OCT parameters and improve visual acuity in patients with subfoveal exsudative AMD, contributing to subjective confidence and consequently compliance with a long term treatment and follow up.

\begin{abstract}
Abbreviations
AMD: age-related macular degeneration; BCVA: best-corrected visual acuity; CNV: choroidal neovascularization; ETDRS: early treatment diabetic retinopathy study; FA: fluorescein angiography; Fab: monoclonal antibody fragment; IOL: intraocular lens; LogMAR: logarithm of minimum angle resolution; NEI-VFQ-25: national eye institute visual functioning questionnaire; PED: pigment epithelial detachment; SD-OCT: spectral domain optical coherence tomography; VEGF: vascular endothelial growth factor.
\end{abstract}

\section{Authors' contributions}

Conduct of the study according to approved protocol, collection of the data and preparation, review or approval of the manuscript (N.A.A., O.F.S.). Both authors read and approved the final manuscript.

\section{Acknowledgements}

Lais Bomediano de Souza, Study Coordinator (SC) of this clinical research under the supervision of the authors.

\section{Competing interests}

The authors declare that they have no competing interests.

\section{Consent for publication}

Written informed consent was obtained from each study participants for participation in the study and for publication of this report "Early changes in macular optical coherence tomography parameters after Ranibizumab intravitreal injection in patients with exsudative age-related macular degeneration". The study and informed consent were approved by the local ethics committee at Medical School of the State University of Campinas.

\section{Ethics approval and consent to participate}

This study was approved by the local committee at Medical School of the State University of Campinas, as well as the consent form (CAEE 00815012.7.0000.5404).

\section{Funding}

This academic study is financially supported by Novartis. The authors do not receive any reimbursement or financial benefits and declare that they have no competing interests. Novartis played no role in the design, methods, data management or analysis or in the decision to publish.

\section{Publisher's Note}

Springer Nature remains neutral with regard to jurisdictional claims in published maps and institutional affiliations.

Received: 3 November 2017 Accepted: 9 January 2018

Published online: 05 February 2018

\section{References}

1. Klein R, Klein BE, Linton KL. Prevalence of age-related maculopathy. The Beaver Dam Eye Study. Ophthalmology. 1992;99(6):933-43.
2. Congdon N, O'Colmain B, Klaver BB, et al. Causes and prevalence of visual impairment among adults in the United States. Eye diseases prevalence research group. Arch Ophthalmol. 2004;122(4):477-85.

3. Ferris FL 3rd, Fine SL, Hyman L. Age-related macular degeneration and blindness due to neovascular maculopathy. Arch Ophthalmol. 1984;102(11):1640-2.

4. Solomon SD, Lindsley K, Vedula SS, Krzystolik MG, Hawkins BS. Antivascular endothelial growth factor for neovascular age-related macular degeneration. Cochrane Database Syst Rev. 2014;29(8):CD005139.

5. Hernandez-Pastor $L$, Ortega A, Garcia-Layana A, Giraldez J. Ranibizumab for neovascular age-related macular degeneration. Am J Health Syst Pharm. 2008;65(19):1805-14.

6. Meyer CH, Holz FG. Preclinical aspects of anti-VEGF agents for the treatment of wet AMD: ranibizumab and bevacizumab. Eye. 2011;25(6):661-72.

7. CATT Research Group. Ranibizumab and bevacizumab for neovascular age-related macular degeneration. N Engl J Med. 2011;364(20):1897-908.

8. Do DV, Gower EW, Cassard SD, et al. Detection of new-onset choroidal neovascularization using optical coherence tomography: the AMD DOC Study. Ophthalmology. 2012;119(4):244-7.

9. Do DV. Detection of new-onset choroidal neovascularization. Curr Opin Ophthalmol. 2013;24(3):244-7.

10. Sayanagi K, Sharma S, Yamamoto T, Kaiser PK. Comparison of spectraldomain versus time-domain optical coherence tomography in management of age-related macular degeneration with Ranibizumab. Ophthalmology. 2009;116(5):947-55.

11. Leung CK, Cheung CY, Weinreb RN, et al. Comparison of macular thickness measurements between time domain and spectral domain optical coherence tomography. Invest Ophtalmol Vis Sci. 2008;49:4893-7.

12. Madureira RF. Avaliação da qualidade de vida pelo questionário NElVFQ-25 nos pacientes portadores da síndrome de Vogt-Koyanagi-Harada. Rio de Janeiro: Faculdade de Medicina, Universidade Federal do Rio de Janeiro; 2005

13. Kaiser PK, Blodi BA, Shapiro H, Achava NR. MARINA Study Group. Angiographic and optical coherence tomographic results of the MARINA study of ranibizumab in neovascular age-related macular degeneration. Ophthalmology. 2007;114(10):1868-75.

14. Rosenfeld PJ, Brown DM, Heier JS, Boyer DS, Kaiser PK, Chung CY. Kim RY for the MARINA study group. Ranibizumab for neovascular age-related macular degeneration. N Engl J Med. 2006;355(14):1419-31.

15. Mangione CM, Lee PP, Gutierrez PR, Spritzer K, Berry S, Hays RD. National Eye Institute Visual Function Questionnaire Field Test Investigators. Development of the 25-intem national eye institute Visual Function Questionnaire. Arch Ophthalmol. 2001;119(7):1050-8.

16. Stewart Michael W MD. Predicted biologic activity of intravitreal bevacizumab. Retina. 2007:27(9):1196-200.

17. Stewart MW, Rosenfeld PJ, Penha FM, Wang F, Yehoshua Z, Bueno-Lopez PF. Pharmacokinetic rationale for dosing every 2 weeks versus 4 weeks with intravitreal ranibizumab, bevacizumab, and aflibercept (vascular endothelial growth factor Trap-eye). Retina. 2012;32(3):434-57.

18. Krohne TU, Liu Z, Holz FG, Meyer CH. Intraocular pharmacokinetics of ranibizumab following a single intravitreal injection in humans. Am J Ophthalmol. 2012;154(4):682-686.e2. https://doi.org/10.1016/j. ajo.2012.03.047

19. Heier JS, Antoszyk AN, Pavan PR, Leff SR, Rosenfeld PJ. Ranibizumab for treatment of neovascular age-related macular degeneration: a phase I/II multicenter, controlled, multidose study. Ophthalmology. 2006;113(4):633.e1-4

20. Novais EA, Badaro E, Hirai FE, et al. Daily Optical Coherence Tomography Examinations after First Antivascular Endothelial Growth Factor Injections: An Interventional Case Series. J Ophthalmol. 2016;2016:6971831. 